\title{
NCCN Guidelines Update: Evolving Radiation Therapy Recommendations for Breast Cancer
}

\author{
Presented by Kilian E. Salerno, MD
}

\section{Abstract}

In the NCCN Clinical Practice Guidelines in Oncology (NCCN Guidelines) for Breast Cancer, among adjuvant radiotherapy options for wholebreast irradiation after breast-conserving surgery, hypofractionation is preferred. For the use of accelerated partial-breast irradiation, the NCCN Guidelines have adopted the updated definition of "suitability" used by the American Society for Radiation Oncology. Regional nodal irradiation is indicated-either in the setting of breast-conserving surgery or after mastectomy-for women with $\geq 4$ positive nodes and should be strongly considered for 1 to 3 positive lymph nodes and select patients with node-negative disease deemed at high risk for recurrence.

J Natl Compr Canc Netw 2017;15(5.5):682-684

In the most recent NCCN Clinical Practice Guidelines in Oncology (NCCN Guidelines) for Breast Cancer, there were updates pertaining to various radiation treatment options, including whole-breast radiotherapy (RT), accelerated partial-breast irradiation (APBI), or regional nodal irradiation (RNI), said Kilian E. Salerno, MD, Associate Professor, Radiation Oncology, and Director, Breast Radiation and Soft Tissue/Melanoma Radiation, Roswell Park Cancer Institute, and a member of the NCCN Breast Cancer Panel.

\section{Adjuvant RT After Breast-Conserving Surgery}

In short, the NCCN recommendations for RT of invasive breast cancer after breast-conserving surgery include RT to the whole breast, with or without a boost; $\mathrm{APBI}$ in select patients; and omission of RT in select patients.

The NCCN recommendations on special considerations for use of breast conservation include 2 new category 2B updates on contraindications for use of RT

Presented by Kilian E. Salerno, MD, Breast Radiation and Soft Tissue/ Melanoma Radiation, Roswell Park Cancer Institute.

Dr. Salerno has disclosed that she has no financial interests, arrangements, affiliations, or commercial interests with the manufacturers of any products discussed in this article or their competitors.

Correspondence: Kilian E. Salerno, MD, Roswell Park Cancer Institute,

Elm \& Carlton Streets, Buffalo, NY 14263

E-mail: kilian.salerno@roswellpark.org after breast-conserving surgery in patient subsets. An absolute contraindication for the use of radiation is the presence of a homozygous ATM mutation. Patients with Li-Fraumeni syndrome have been included as a relative contraindication to radiation.

The NCCN recommendations on appropriate margins for breast-conserving surgery have been updated for both ductal carcinoma in situ (DCIS) and invasive breast cancer in patients who will receive wholebreast irradiation. The appropriate surgical margins for lumpectomy are now defined as $2 \mathrm{~mm}$ for DCIS and "no tumor on ink" for invasive carcinoma. These recommendations are not applicable for APBI or patients who have received neoadjuvant chemotherapy.

\section{Hypofractionation: Preferred for Whole-Breast RT}

For most women receiving whole-breast irradiation, hypofractionation is preferred by the NCCN panel. Hypofractionation is a shorter treatment course using slightly higher doses per fraction (>2 Gy/fraction) and fewer fractions compared with conventional fractionation (1.8-2.0 Gy/fraction). According to Dr. Salerno, for many patients, hypofractionation achieves results that are "at least equivalent to or better than" those seen with conventional fractionation in terms of local control, breast cosmesis, and toxicity. The recommendation 
is based on long-term results from the Canadian tri$\mathrm{al}^{1}$ and the START-B trial. ${ }^{2}$

"We hope to move away from the concept of conventional fractionation being considered standard," she said, "because I would argue that for whole-breast RT, hypofractionation is the standard as well."

Hypofractionation, however, is not generally to be used in the postmastectomy setting or when treating regional nodes.

\section{Updated Statement Regarding APBI}

For APBI, the NCCN panel accepts and has adopted the updated 2016 version of the guidelines issued by the American Society for Radiation Oncology (ASTRO) $)^{3}$ for determining suitability:

- Patients aged $\geq 50$ years with invasive ductal carcinoma measuring $\leq 2 \mathrm{~cm}$ (T1 disease) with negative margin widths of $\geq 2 \mathrm{~mm}$, no lymphovascular invasion, estrogen receptor-positive status, and negative for the BRCA mutation.

- Patients with low/intermediate nuclear grade, screen-detected DCIS measuring $\leq 2.5 \mathrm{~cm}$ with negative margin widths of $\geq 3 \mathrm{~mm}$.

"Of particular focus, within the suitable group, the age criterion has been lowered to $\geq 50$ years from 60 years," she said. "Also, DCIS was previously in the 'cautionary' group, but has now moved into the suitable group, provided certain criteria are met."

The new DCIS criteria were adopted from the RTOG 9804 trial in women with "good-risk" DCIS. ${ }^{4}$ At 7 years, local failure rates were $6.7 \%$ without RT and $0.9 \%$ with RT.

\section{Omission of RT}

Omission of RT altogether is an acceptable option in select women with a lower risk for recurrence. Based largely on the long-term results from the CALGB $9343^{5}$ trial, the guidelines reflect that omission of RT may be appropriate in women aged $\geq 70$ years with estrogen receptor-positive, clinically node-negative T1 tumors who will receive adjuvant endocrine therapy. Similar results were reported on the PRIME II, ${ }^{6}$ and there are a number of clinical trials ongoing for omission of RT in low-risk women as defined by recurrence scores.

\section{Who Needs RNI?}

The guidelines regarding RNI in the treatment of patients with node-positive and/or high-risk, nodenegative disease have been updated on the basis of many studies, including the Early Breast Cancer Trialists' Collaborative Group (EBCTCG) meta-analysis and the MA.20 and EORTC 22922 trials.

In EBCTCG's analysis of 22 randomized trials of 8,135 patients undergoing mastectomy and axillary dissection, RT reduced locoregional recurrences, overall recurrences, and breast cancer mortality in women with $\geq 4$ positive nodes or 1 to 3 positive nodes. ${ }^{7}$ The absolute difference in breast cancer mortality was $7.9 \%$ at 20 years. Similar effects were seen for those with 1 positive node. Benefit was not seen in patients with node-negative disease.

After 10 years of follow-up in MA.20 and in EORTC 22922, ${ }^{9}$ RNI was associated with improvement in locoregional and disease-free survival and lower breast cancer mortality, but not overall survival. These 2 studies evaluated similar approaches to RNI, although their patient populations were different.

A focused guideline update on postmastectomy RT (PMRT) was recently published addressing the role of radiation in patients with $\mathrm{T} 1-2$ breast cancer and 1 to 3 positive nodes. ${ }^{10}$ The panel recognized that use of PMRT reduces locoregional recurrences and improves breast cancer mortality. Treatment recommendations for use of PMRT or not should include clinical judgement and assessment of individual risk.

"We have all these trials that interplay and influence our thinking about what to do for node-positive disease; we have to interpret them and reconcile the differences in trying to determine individual treatment recommendations," Dr. Salerno said. Clinicians should determine the individual patient's risk for recurrence and whether the patient fits the eligibility criteria of these studies.

RNI should be used in patients with $\geq 4$ positive nodes or locally advanced tumors, should be strongly considered for patients with 1 to 3 positive nodes, and may also be considered for select patients with node-negative disease at high risk. Clinicians should assess individual patient risk for recurrence and apply RNI accordingly, considering age, life expectancy, comorbidities, tumor size, lymphovascular invasion, number and size of positive lymph nodes, response to 
neoadjuvant chemotherapy, extent of residual disease, management of the axillae, and intrinsic tumor type.

RNI includes treatment of the supraclavicular area, infraclavicular region, internal mammary nodes, and any part of the axillary bed considered at risk.

\section{RT After Neoadjuvant Treatment}

Following neoadjuvant chemotherapy, indications for RT should be guided by maximal disease stage from either prechemotherapy tumor characteristics at diagnosis or postchemotherapy pathology results. Patients with residual nodal disease after neoadjuvant systemic therapy should receive RNI.

Recurrence risk may be lower for patients who experience a pathologic complete response, but at this time, this does not negate the need for RT if indications for treatment were present at diagnosis.

Studies evaluating management of the regional nodes in the setting of neoadjuvant chemotherapy are ongoing, including NSABP B-51/RTOG 1304 and the Alliance 011202 trials. Clinicians are encouraged to enroll patients on these studies.

\section{Optimizing RT Treatment}

Numerous treatment modalities and techniques are available for breast radiation with the intent to provide homogenous dose to targets and minimize toxicity to adjacent normal tissues. "Radiation oncologists have a broad spectrum of options," Dr. Salerno noted, including prone positioning in select patients, use of respiratory control techniques, and CT-based treatment planning.

\section{References}

1. Whelan TJ, Pignol JP, Levine MN, et al. Long-term results of hypofractionated radiation therapy for breast cancer. $N$ Engl J Med 2010;362:513-520.

2. Haviland JS, Owen JR, Dewar JA, et al. The UK Standardisation of Breast Radiotherapy (START) trials of radiotherapy hypofractionation for treatment of early breast cancer: 10-year follow-up results of two randomized controlled trials. Lancet Oncol 2013;14:1086-1094.

3. Correa C, Harris EE, Leonardi MC, et al. Accelerated partial breast irradiation: executive summary for the update of an ASTRO evidencebased consensus statement. Pract Radiat Oncol 2017;7:73-79.

4. McCormick B, Winter K, Hudis C, et al. RTOG 9804: a prospective randomized trial for good-risk ductal carcinoma in situ comparing radiotherapy with observation. J Clin Oncol 2015;33:709-715.

5. Hughes KS, Schnaper LA, Bellon JR, et al. Lumpectomy plus tamoxifen with or without irradiation in women age 70 years or older with early breast cancer: long-term follow-up of CALGB 9343. J Clin Oncol 2013;31:23822387.

6. Kunkler IH, Williams LJ, Jack WJ, et al. Breast-conserving surgery with or without irradiation in women aged 65 years or older with earl breast cancer (PRIME II): a randomized controlled trial. Lancet Oncol 2015;16:266-273

7. EBCTCG (Early Breast Cancer Trialists' Collaborative Group), McGale P, Taylor C, et al. Effect of radiotherapy after mastectomy and axillary surgery on 10-year recurrence and 20-year breast cancer mortality: meta-analysis of individual patient data for 8135 women in 22 randomised trials. Lancet 2014:383:2127-2135.

8. Whelan TJ, Olivotto IA, Parulekar WR, et al. Regional nodal irradiation in early-stage breast cancer. N Engl J Med 2015;373:307-316.

9. Poortmans PM, Collette S, Kirkove C, et al. Internal mammary and medial supraclavicular irradiation in breast cancer. N Engl J Med 2015;373:317327.

10. Recht A, Comen EA, Fine RE, et al. Postmastectomy radiotherapy: an American Society of Clinical Oncology, American Society for Radiation Oncology, and Society of Surgical Oncology focused guideline update. J Clin Oncol 2016;34:4431-4442. 